\title{
Histone acetylation regulates the expression of genes involved in worker reproduction and lifespan in the ant Temnothorax rugatulus
}

\author{
Marina Choppin ${ }^{1}$, Barbara Feldmeyer ${ }^{2}$, and Susanne Foitzik ${ }^{3}$ \\ ${ }^{1}$ Johannes Gutenberg Universitat Mainz \\ ${ }^{2}$ Senckenberg Biodiversity and Climate Research Centre (BiK-F) \\ ${ }^{3}$ University of Mainz
}

April 29, 2021

\begin{abstract}
In insect societies, the queen monopolizes reproduction while workers perform tasks such as brood care or foraging. Queen loss leads to ovary development and lifespan extension in workers from many ants. However, the underlying molecular mechanisms of this phenotypic plasticity remain unclear. Recent studies highlight the importance of epigenetics in regulating plastic traits in social insects. We investigated the role of histone acetylation in the regulation of worker reproduction in the ant Temnothorax rugatulus. We removed queens from their colonies to induce worker fecundity, and either fed workers with chemical inhibitors of histone acetylation (C646), deacetylation (Trichostatin A), or the solvent (DMSO) as control. We monitored worker number for six weeks after which we assessed ovary development and sequenced fat body mRNA. Workers survived better in queenless colonies and developed their ovaries after queen removal in control colonies as expected, but not in colonies treated with chemical inhibitors. Both inhibitors affected gene expression, although the inhibition of histone acetylation using C646 influenced the expression of more genes with immunity, fecundity, and longevity functionalities. Interestingly, these C646-treated workers shared many upregulated genes with infertile workers from queenright colonies. We also identified one gene with antioxidant properties commonly downregulated in infertile workers from queenright colonies and both C646 and TSA-treated workers from queenless colonies. Our results indicate that histone acetylation is involved in the molecular regulation of worker reproduction and lifespan, and thus point to an important role of histone modifications in modulating phenotypic plasticity of life history traits in social insects.
\end{abstract}

Histone acetylation regulates the expression of genes involved in worker reproduction and lifespan in the ant Temnothorax rugatulus

Running title: Epigenetic regulation of ant worker fecundity

Marina Choppin ${ }^{1 *}$, Barbara Feldmeyer ${ }^{2}$ and Susanne Foitzik ${ }^{1}$

${ }^{1}$ Institute of Organismic and Molecular Evolution, Johannes Gutenberg University, Mainz, Germany

${ }^{2}$ Senckenberg Biodiversity and Climate Research Centre (SBiK-F), Molecular Ecology, Senckenberg, Frankfurt, Germany

*contact author:mchoppin@uni-mainz.de

\section{Abstract}


In insect societies, the queen monopolizes reproduction while workers perform tasks such as brood care or foraging. Queen loss leads to ovary development and lifespan extension in workers from many ants. However, the underlying molecular mechanisms of this phenotypic plasticity remain unclear. Recent studies highlight the importance of epigenetics in regulating plastic traits in social insects. We investigated the role of histone acetylation in the regulation of worker reproduction in the ant Temnothorax rugatulus . We removed queens from their colonies to induce worker fecundity, and either fed workers with chemical inhibitors of histone acetylation (C646), deacetylation (Trichostatin A), or the solvent (DMSO) as control. We monitored worker number for six weeks after which we assessed ovary development and sequenced fat body mRNA. Workers survived better in queenless colonies and developed their ovaries after queen removal in control colonies as expected, but not in colonies treated with chemical inhibitors. Both inhibitors affected gene expression, although the inhibition of histone acetylation using C646 influenced the expression of more genes with immunity, fecundity, and longevity functionalities. Interestingly, these C646-treated workers shared many upregulated genes with infertile workers from queenright colonies. We also identified one gene with antioxidant properties commonly downregulated in infertile workers from queenright colonies and both C646 and TSA-treated workers from queenless colonies. Our results indicate that histone acetylation is involved in the molecular regulation of worker reproduction and lifespan, and thus point to an important role of histone modifications in modulating phenotypic plasticity of life history traits in social insects.

Keywords: gene regulation, epigenetics, fecundity, longevity, ants

\section{Introduction}

The societies of social insects exhibit a reproductive division of labor where the queen reproduces, whereas workers perform all other tasks including brood care, nest defense, and foraging (Hölldobler \& Wilson, 1990). Workers thus sacrifice their own reproduction. This evolutionary incongruity is commonly explained by Hamilton's inclusive fitness theory, which states that genes of sterile altruists can be transmitted indirectly to the next generation by helping closely related reproductives (Hamilton, 1964). The proximate mechanisms underlying the maintenance of worker sterility in social insect colonies have been extensively investigated as well. Worker reproduction is regulated via chemical signals emitted by the queen or her brood (Endler et al., 2004; Matsuura et al., 2010; Van Oystaeyen et al., 2014), or through social control meditated by the queen or workers themselves (Foster \& Ratnieks, 2000; Oldroyd et al., 2001; Ruhland, Moulin, Choppin, Meunier, \& Lucas, 2020). However, in rare cases, social insect workers circumvent those restraints even in queenright colonies and successfully gain direct fitness benefits by laying haploid eggs developing into males (Beekman \& Oldroyd, 2008; Giehr, Wallner, et al., 2020). Conversely, worker reproduction is frequently found in queenless colonies. Queen loss first induces fights among workers, who establish a new reproductive hierarchy, develop their ovaries, and start laying haploid eggs (Giehr, Senninger, Ruhland, \& Heinze, 2020; Heinze, 2008; Monnin \& Peeters, 1999). Reproduction has strong effects on the physiology and immunity of workers, who can become more resistant to oxidative stress and often live longer (dos Santos Conceição Lopes, Campbell, \& Contrera, 2020; Kohlmeier et al., 2017; Majoe, Libbrecht, Foitzik, \& Nehring, 2021; Negroni, Segers, Vogelweith, \& Foitzik, 2020). These positive effects of reproduction have been linked to the activation of signaling pathways such as insulin/insulin-like growth factor (IIS), the target of rapamycin (mTOR), and the alpha-ketoglutarate (alpha-KG) (Negroni, Macit, Stoldt, Feldmeyer, \& Foitzik, 2021). Indeed, gene expression changes profoundly in workers after queen loss in social wasps (Taylor, Cini, Sumner, Wyatt, \& Reuter, 2021), honeybees (Cardoen et al., 2011), and ants (Negroni et al., 2021; Wurm, Wang, \& Keller, 2010) and in various tissues from the brain to the fat body. Similarly, gene expression differs between reproductive and sterile bumblebee workers (Marshall, Lonsdale, \& Mallon, 2019). Worker fecundity thus appears to be a highly plastic trait positively linked to lifespan (Heinze \& Schrempf, 2008). This opens up exciting new avenues to study the molecular regulation of plasticity in fecundity and longevity in social insect workers (Monroy Kuhn \& Korb, 2016). Indeed, the transcriptomic changes linked to worker reproduction have been well characterized, while the underlying gene regulatory mechanisms remain largely unexplored.

Epigenetic mechanisms including DNA methylation and histone modifications have been proposed to play 
a major role in the extraordinary phenotypic plasticity exhibited by social insects (Bonasio, 2012, 2014; Herb, 2014; Maleszka, 2016; Vaiserman, 2015; Vaiserman, Lushchak, \& Koliada, 2018; Yan et al., 2015). In Carpenter ants, histone modifications have been associated with behavioral differences between major and minor ant workers (Glastad et al., 2019; Simola et al., 2016, 2013) and worker polymorphism (Alvarado, Rajakumar, Abouheif, \& Szyf, 2015). Histone acetylation has also been associated with the ability of workers to adjust to new daily rhythms (Libbrecht, Nadrau, \& Foitzik, 2020). Besides, there is growing evidence for the role of histone modifications in caste differentiation. In honey bees, queen development is largely controlled by royal jelly, a secretion that has histone deacetylase inhibitor (HDACi) activity (Spannhoff et al., 2011). Moreover, caste-determined female larvae exhibit genome-wide differences in histone acetylation and methylation patterns, which are linked to caste-specific gene expression (Wojciechowski et al., 2018). Besides, the transition from workers to reproductive gamergates has been associated with changes in gene expression related to epigenetic pathways in the ant Harpegnathos saltator (Bonasio et al., 2010).

In this study, we use the ant Temnothorax rugatulus to investigate the role of histone acetylation in the regulation of reproduction and lifespan in queenless workers. This common Myrmicine ant builds small nests of 50 to 2000 workers with one to several queens and evolved two queen morphs, the large macrogynes, and the small microgynes, associated with alternative reproductive strategies (Choppin et al., 2021; Rüppell, Heinze, \& Hölldobler, 1998, 2001). Queens can live over ten years and their gene expression in the brain and fat body changes with age (Negroni, Foitzik, \& Feldmeyer, 2019). Following queen loss, T. rugatulus workers are known to develop their ovaries, start laying haploid eggs, live longer, and show transcriptomic changes in the fat body, a physiologically active tissue (Negroni et al., 2021, 2020). Here, we asked whether histone acetylation is required for workers to plastically respond to queen loss by altering their ovary development and associated gene expression. We used queen removal to induce fecundity in workers while feeding them with chemical inhibitors of histone acetylation (C646) or deacetylation (TSA). Based on previous studies, we predicted that workers would develop ovaries, survive better, and show transcriptomic changes following queen removal. If histone acetylation does play a role in the regulation of worker reproduction and lifespan, we expected the chemical inhibitors C646 and TSA to negatively impact worker ovary development following queen removal, and to alter the regulation of fecundity and longevity genes, preventing workers in queenless colonies to reproduce and live longer.

\section{Materials and Methods}

\section{Ant collection and maintenance}

Temnothorax rugatulus ants are distributed throughout the western part of North America and reside in high elevation coniferous forests, under stones or in rock crevices. In August 2018, we collected colonies from nine different locations in the Chiricahua Mountains (Arizona, USA, Table S1). In the laboratory, each colony was kept in a three-chambered box $(9.7 \times 9.7 \times 2.9 \mathrm{~cm})$ covered with a lid and containing an artificial nest made of a plastic insert between two glass slides covered by a red foil to block the light. The colonies were maintained at $21^{\circ} \mathrm{C}$ and $70 \%$ humidity with a $12: 12$ light:dark cycle. They were fed weekly with half a cricket and a drop of honey and were provided with water ad libitum.

\section{Colony monitoring}

We selected 90 monogynous colonies with 54 to 100 workers and reduced worker number to 50 per colony. Colonies were then moved to a climate chamber at $25^{\circ} \mathrm{C}$ and $70 \%$ humidity with a $12: 12$ light:dark cycle for two weeks. Before starting the experiment, colonies were randomly assigned to one of five experimental groups with a total of 18 colonies per group (Table 1 ).

On the first day of the experiment, we removed all eggs, pupae, and males and adjusted the number of larvae to five per colony. Queens from the queen removal groups were removed and returned to their natal colonies. Colonies were fed with either the solvent dimethyl sulfoxide (DMSO; Carl Roth) only, the inhibitor of histone acetylation C646 which targets the histone acetyltransferase p300 (50 $\mu \mathrm{M}$ in DMSO; Sigma-Aldrich) (Bowers et al., 2010), the inhibitor of histone deacetylation Trichostatin A (TSA) known as class I and class II histone 
deacetylase inhibitor (50 $\mu \mathrm{M}$ in DMSO; Sigma-Aldrich) (Yoshidas, 1990), or a combination of C646 and TSA (both $50 \mu \mathrm{M}$ in DMSO). All preparations were diluted in $0.102 \mathrm{~g} / \mathrm{mL}$ sucrose solution. The ants were fed for six weeks every other day with $15 \mu \mathrm{L}$ of fresh solution per colony (Figure S1). Additionally, each colony received half a cricket every other day and water ad libitum. Once a week, we anesthetized all colonies with $\mathrm{CO}_{2}$. We removed and counted the eggs in queenless colonies to get precise numbers of worker-laid eggs. Once every two weeks, we counted all colony members (queens if applicable, workers, eggs, larvae, and pupae) in colonies from all groups.

We tested the effect of queen removal on worker survival by comparing worker number over time between the groups "queenright" and "queenless" using a linear mixed-effects model (LMM) with the package "lme4" (Bates, Mächler, Bolker, \& Walker, 2015). In queenless colonies, we investigated the effect of treatment (DMSO, DMSO+C646, DMSO+TSA, and DMSO+C646/TSA) on worker number in interaction with time using a similar model. Colony identification (ID) was used as a random factor in both models to account for inter-colony variability. We assessed the fit of our LMMs using visual inspections of the residual distributions. Treatment effect on egg production at week six was analyzed using a generalized linear mixed-effects model (GLMM, binomial family) with egg production as a binary variable. Our GLMM was tested for overdispersion using the package "DHARMa" (Hartig, 2020). All statistical analyses were conducted in R v3.5.1 (R Core Team, 2020).

\section{Dissections, RNA extraction, and fecundity measures}

After six weeks, we selected a subset of colonies for dissections (SI Methods). For each colony, we isolated all the workers close to the brood in a Petri dish and dissected them on ice in a drop of a sterile saline solution until two workers with developed ovaries were found ( 2 to 14 workers dissected per colony). From these two workers, we cleaned the ovaries and took pictures for fecundity measurements using a stereomicroscope (details below). The fat bodies, including the first cuticle plate of the gaster, were collected from the two workers and pooled in the same Eppendorf tube containing $50 \mu \mathrm{l}$ of TRIzol (Thermofisher) for further RNAsequencing. Tissue collection took less than 10 minutes. The samples were flash-frozen in liquid nitrogen and preserved at $-80^{\circ} \mathrm{C}$. Before the RNA extraction of each sample, we crushed the fat bodies with a pestle, added $50 \mu \mathrm{l}$ of phenol:chloroform:isoamyl alcohol (25:24:1) (Carl Roth), mixed manually, and centrifuged at $1200 \mathrm{xg}$ for 15 minutes. Afterwards, the upper phase was transferred to a new tube and mixed with $25 \mu \mathrm{l}$ of ethanol $100 \%$ (Carl Roth). RNA was then extracted using a NucleoSpin RNA XS kit (Macherey-Nagel). After quantity and quality control, 20 samples were sent for sequencing (Table 1). Library preparation was conducted following the standard protocol of BGI (Hongkong), which sequenced 150 bp paired-end reads on an Illumina Hiseq X ten.

We measured ovariole length and counted the number of white eggs (i.e., eggs in development) and yellow bodies in the ovaries using the Leica software LAS v4.5. Yellow bodies are an indication of recent egg laying in ants (Cini, 2014; Heinze, Hölldobler, \& Trenkle, 1995; Peeters \& Tinaut, 2014). We analyzed the effects of queen removal and treatment (DMSO, DMSO+C646, DMSO+TSA, DMSO+C646/TSA) on worker ovariole length using LMMs. We used GLMMs (binomial family) to test for effects of queen removal and treatment on the presence of white eggs and yellow bodies in the ovaries. Colony ID was used as a random factor to account for inter-colony variability. The models' fit was assessed as described above.

\section{Gene expression analysis}

Raw reads were trimmed with Trimmomatic v0.39 (Bolger, Lohse, \& Usadel, 2014) (Table S2) and quality checked using FastQC v0.11.7 (Andrew, 2010). The paired reads were then mapped against the Temnothorax rugatulus draft genome (Jongepier et al. unpub.) using HISAT2 v2.1.0 (Kim, Langmead, \& Salzberg, 2015) (Table S2). We converted and sorted the output files using SAMtools v1.7 (Li et al., 2009) and obtained a quality report from Qualimap v2.2.1 (Okonechnikov, Conesa, \& García-Alcalde, 2016). A genome guided transcriptome assembly was created using StringTie v2.1.3 (Pertea et al., 2015) and transcript sequences were extracted using GffRead v0.11.8 on the merged GTF file. Transcriptome quality was assessed using TransRate v1.0.3 (Smith-Unna, Boursnell, Patro, Hibberd, \& Kelly, 2016). Transcripts with an Open Reading Frame 
$(\mathrm{ORF})<100$ bp were removed and the Python script "prepDE.py" from the online StringTie Manual was used to generate the gene count matrix (Table S3).

We assessed the effect of queen removal on worker gene expression by comparing the groups "queenless" and "queenright". Then, we tested the effects of the chemical inhibition of histone acetylation and deacetylation by first comparing the groups "queenless" to "queenless+C646", and then "queenless" to "queenless+TSA". To avoid factitious DESeq2 results and for each comparison, we first filtered the gene count matrix so at least $70 \%$ of samples had a read count of ten or more reads per gene in at least one experimental group. We additionally plotted the maximum cook distance against the average gene expression per sample to identify and remove putative outliers. We used the filtered count matrix (Table S4) to perform the differential gene expression analysis using DESeq2 (Love, Huber, \& Anders, 2014) setting an adjusted p-value $<0.05$ as significance threshold. We plotted principal component analyses (PCAs) with all genes using the package "ggplot2" (Wickham, 2010) to assess the group-based clustering of our samples (Figure S2, S3, and S4). We created heatmaps with the package "pheatmap" (Kolder, 2012) to visualize expression differences and clustering between samples.

To annotate transcripts we conducted a BlastX homology search with BLAST v2.10.1+ (Altschul, Gish, Miller, Myers, \& Lipman, 1990) using the non-redundant invertebrate protein database from NCBI (May 2020) and only considered hits with an E-value $<10^{-5}$ (SI Methods). We combined the blast annotations with gene information from UniProt (www.uniprot.org) . We used TransDecoder v5.5.0 (Haas et al., 2013) to translate nucleotide sequences into amino-acid sequences and then ran InterProScan v5.45-80.0 (Quevillon et al., 2005) to obtain Gene Ontology (GO) term annotations. Then, we performed a GO term enrichment analysis using the R package "topGO" (Alexa \& Rahnenführer, 2020) with the algorithm "weight01" (SI Methods). We conducted the GO enrichment analysis separately for upregulated and downregulated genes in the groups compared to the queenless control. Statistical significance was given using Fischer exact tests. We extracted the overlap of upregulated and downregulated genes between the groups "queenright", "queenless+C646" and "queenless+TSA" and assessed whether the overlap size between two groups was larger than expected by chance by resampling random gene lists (500 iterations). Finally, we plotted expression levels (i.e., normalized read counts) of genes of interest using "plotCounts" from DESeq2.

\section{Results}

\section{Effects of queen removal}

Worker number and fecundity

Worker number decreased over time $\left(\mathrm{X}^{2}=441.663, \mathrm{df}=2, \mathrm{p}<0.001\right)$, although less strongly in queenless colonies $\left(\mathrm{X}^{2}=9.723, \mathrm{df}=2, \mathrm{p}=0.008\right.$; Figure $\left.\mathrm{S} 5\right)$. Workers from queenless colonies had longer ovarioles $\left(\mathrm{X}^{2}=30.578, \mathrm{df}=1, \mathrm{p}<0.001 ;\right.$ Figure $\left.1 \mathrm{~A}\right)$, were more likely to have yellow bodies $\left(\mathrm{X}^{2}=9.588, \mathrm{df}=1, \mathrm{p}\right.$ $=0.002$; Figure $1 \mathrm{~B})$, and also tentatively more likely to have white eggs in their ovaries $\left(\mathrm{X}^{2}=2.828, \mathrm{df}=\right.$ $1, \mathrm{p}=0.093 ;$ Figure $\mathrm{S} 6$ ).

Gene expression and functional enrichment

We found 346 differentially expressed genes (DEGs) between workers from the groups "queenless" and "queenright" (Table S5A-B), among which 206 were upregulated and 140 downregulated in the queenright group. The samples clearly clustered according to treatment in the heatmap based on all DEGs (Figure 1C). The enrichment analysis revealed that workers in presence of their queen downregulated genes related to five functions including "innate immune response" (Figure 1D, Table S6B). This is reflected by the presence of multiple immune genes in our top 15 downregulated genes in the queenright group including "FK506-binding protein 2 isoform X1" or "chymotrypsin-2-like". Also, the gene coding for "vitellogenin-1-like" (Figure 2) was found to be downregulated in queenless workers. Queen presence affected the expression of many regulatory genes as well, such as transcription factors including "zinc finger protein 454-like".

Effects of chemical inhibitors 
Worker number, egg production, and fecundity

Here we focus on queenless colonies that were either fed with DMSO only (control) or additionally treated with C646, TSA or both inhibitors. Again, worker number generally decreased over time $\left(\mathrm{X}^{2}=719.732\right.$, $\mathrm{df}=2, \mathrm{p}<0.001)$, but irrespective of treatment $\left(\mathrm{X}^{2}=7.493, \mathrm{df}=6, \mathrm{p}=0.278\right.$; Figure $\left.\mathrm{S} 7\right)$. After six weeks, $23 \%$ of colonies had eggs and the presence of eggs was unaffected by treatment $\left(\mathrm{X}^{2}=2.521\right.$, $\mathrm{df}=$ $3, \mathrm{p}=0.472$; Figure S8). However, workers treated with the epigenetic inhibitors had shorter ovarioles $\left(\mathrm{X}^{2}\right.$ $=11.569, \mathrm{df}=3, \mathrm{p}=0.009$; Figure $3 \mathrm{~A})$ and a smaller proportion of treated workers had yellow bodies in their ovaries $\left(\mathrm{X}^{2}=9.721, \mathrm{df}=3, \mathrm{p}=0.021\right.$; Figure $\left.3 \mathrm{~B}\right)$ compared to control workers. More precisely, C646-treated workers exhibited shorter ovarioles $\left(\mathrm{t}_{\mathrm{C} 646}=-3.290, \mathrm{p}_{\mathrm{C} 646}=0.002\right)$ and were less likely to have yellow bodies in the ovaries $\left(\mathrm{z}_{\mathrm{C} 646}=-2.366, \mathrm{p}_{\mathrm{C} 646}=0.018\right)$ compared to workers fed with DMSO only. In the TSA treatment, fewer workers had yellow bodies in their ovaries compared to control workers $\left(\mathrm{z}_{\mathrm{TSA}}=\right.$ $\left.-2.949, \mathrm{p}_{\mathrm{TSA}}=0.003\right)$, although ovariole length was unaffected by TSA treatment $\left(\mathrm{t}_{\mathrm{TSA}}=-0.891, \mathrm{p}_{\mathrm{TSA}}=\right.$ $0.379)$. None of the inhibitors affected the proportion of workers with white eggs in the ovaries $\left(\mathrm{X}^{2}=5.817\right.$, df $=3, \mathrm{p}=0.121 ;$ Figure S9).

Gene expression and functional enrichment

We found 306 differentially expressed genes between workers from the control group and workers treated with the inhibitor of histone acetylation C646 (Table S5C-D), among which 247 were upregulated and 59 were downregulated in the C646-treated workers. The heatmap revealed that C646 samples clustered well together (Figure 3C). C646-treated workers downregulated genes with longevity functionalities such as "protein I'm not dead yet" also called "Indy" (Rogina \& Helfand, 2013) (Figure 2), or genes with an immune function like "proclotting enzyme-like" (Villanueva-Segura et al., 2020) (Figure 2). Workers fed with C646 also downregulated seven genes related to the synthesis of fatty acids, versus one only in the control. Our enrichment analysis revealed the overrepresentation of five functions associated with downregulated genes of C646-treated workers including "oxidation-reduction process" (Figure 3D, Table S6D).

Between workers from the control group and workers treated with the inhibitor of histone deacetylation TSA, we found 32 differentially expressed genes (Table S5E-F). Only a single gene was upregulated and 31 were downregulated in the TSA-treated workers. The heatmaps created using the 32 DEGs revealed a looser clustering of our samples by group (Figure S10), which is consistent with the weaker effect of TSA treatment on the number of DEGs. Based on the low number of DEGs between the two groups we only found the functions "transposition, DNA-mediated" and "autophagy" significantly overrepresented in the DEGs of the TSA-treated workers (Table S6E-F). Although the TSA treatment also had weaker effects on worker fecundity, we did find the aging and fecundity-associated gene "juvenile hormone acid O-methyltransferaselike isoform X1" (Hartfelder, 2000; Yamamoto, Bai, Dolezal, Amdam, \& Tatar, 2013) downregulated in TSA-treated workers (Figure 2).

\section{Overlapping genes between groups}

As indicated above, the chemical inhibitors of histone acetylation and deacetylation impaired worker ovary development following queen removal. Thus, we asked whether the transcriptomes of inhibitor-treated workers were similar to the ones of infertile workers from queenright colonies. Indeed, between the groups "queenright" and "queenless+C646" we found 82 genes commonly upregulated (Figure 4A, Table S5G) and five genes commonly downregulated (Figure 4B, Table $\mathrm{S} 5 \mathrm{H}$ ) in workers. We additionally found five genes commonly downregulated between the groups "queenright" and "queenless+TSA" (Figure 4B, Table S5I). These three numbers of overlapping genes were higher than expected by chance as evidenced by resampling random gene lists (Figure S11). Among the commonly upregulated genes between workers with a queen and C646treated workers, we found genes associated with circadian rhythm like "circadian clock-controlled protein" (Figure 2). We additionally found many genes associated with digestion like "mucin-5AC-like", "probable salivary secreted peptide" or "silk gland factor 1". Finally, the gene "transketolase-like protein 2 isoform X2" with antioxidant properties (Kovarova et al., 2018; Schenk, Duggleby, \& Nixon, 1998; Xu et al., 2016) was commonly downregulated in all three groups in comparison to the control (Figure 2). 


\section{Discussion}

In this study, we investigated the role of histone acetylation in the regulation of genes associated with worker reproduction and lifespan in the ant Temnothorax rugatulus. We removed queens from their colonies while feeding the workers with chemical inhibitors of histone acetylation (C646) or deacetylation (TSA). Our phenotypic and transcriptomic results confirmed that worker fecundity and survival increased after queen removal, as shown before in this species (Negroni et al., 2021, 2020). However, the ovary development of queenless workers was impaired when treated with the chemical inhibitors. On a molecular level, the inhibition of histone acetylation using $\mathrm{C} 646$ had a stronger effect than the inhibition of histone deacetylation using TSA, as shown by the difference in the number of differentially expressed genes (306 vs 32). Yet, workers from both treatments downregulated many genes related to fecundity, immunity, and longevity, compared to the queenless control. More importantly, a large number of genes were commonly upregulated between workers from queenright colonies (i.e., infertile workers) and C646-treated workers from queenless colonies, pointing to similar transcriptomes. We additionally found one gene with antioxidant properties commonly downregulated between infertile workers and both C646 and TSA-treated workers, in comparison to fertile ants in the DMSO control, which might be linked to the extended survival of these fertile ants.

Ant workers start to reproduce and live longer after the loss of their queen in many ant species (Heinze, 2008; Kohlmeier et al., 2017; Konrad, Pamminger, \& Foitzik, 2012) including our model Temnothorax rugatulus (Negroni et al., 2021, 2020). We took advantage of this plasticity by inducing fecundity in workers using queen removal and confirmed that workers in queenless colonies developed their ovaries, started laying eggs, survived better, and shifted their gene expression in the fat body. Our transcriptomic analysis revealed avitellogenin $(\mathrm{vg})$ gene to be upregulated in workers in the absence of their queen. During social insect evolution, $v g$ genes underwent several duplications followed by diversification and sub-functionalization. Various $v g$ orthologues now take over different functions in behavior (Kohlmeier, Feldmeyer, \& Foitzik, 2018) and physiology, including fecundity (Amdam, Norberg, Hagen, \& Omholt, 2003) and aging (Parker, 2010) in social insects. For example, the expression of conventional vitellogenins is linked to oxidative stress resistance in honeybee workers (Seehuus, Norberg, Gimsa, Krekling, \& Amdam, 2006). We investigated where our "vitellogenin-1-like" copy falls within the vitellogeninphylogeny (Kohlmeier et al., 2018, data not shown) and found that it clusters close to the conventional vitellogenins, indicating an ancestral fecundity function as an egg yolk precursor (Amdam et al., 2003) rather than a derived role, as in worker caste differentiation (Feldmeyer, Elsner, \& Foitzik, 2014; Morandin et al., 2014). Interestingly, a previous study on the effect of queen removal on worker gene expression in T. rugatulus detected a vitellogeninreceptor to be upregulated in fertile, queenless workers (Negroni et al., 2020), pointing to the importance of $v g$-associated pathways for worker reproduction.

Longevity is traded-off with immunity in many organisms due to the cost of an active immune system and the production of reactive oxygen species during immune reactions (DeVeale, Brummel, \& Seroude, 2004; Garschall \& Flatt, 2018). Nevertheless, our results show that the longer-lived, fertile workers from queenless colonies activate genes with an innate immune response functionality, which is consistent with previous findings in Temnothorax rugatulus showing that fertile workers express more immunity genes following an immune challenge, compared to infertile workers (Negroni et al., 2020). Similarly, the highly fertile young queens of T. rugatulus upregulate immune genes in the Toll-pathway (Negroni et al., 2019), which plays a crucial role in insect immunity (Valanne, Wang, \& Rämet, 2011). These findings provide evidence that fecund ants invest in a longer lifespan and immunity concurrently, supporting other studies on social insects indicating that life history trade-offs have shifted during their social evolution (Korb, 2016; Schrempf, Giehr, Röhrl, Steigleder, \& Heinze, 2017).

Interestingly, ant workers treated with chemical inhibitors following queen removal had a lower ovary development and were less likely to exhibit yellow bodies, which provide evidence for egg-laying (Cini, 2014; Heinze et al., 1995; Peeters \& Tinaut, 2014) compared to control workers from queenless nests. This indicates that dynamic changes in histone acetylation might be required for workers to shift to longer-lived, fecund 
phenotypes. In social insects, histone acetylation has previously been linked to various processes including the regulation of foraging behavior and caste determination (Simola et al., 2016, 2013; Spannhoff et al., 2011; Wojciechowski et al., 2018), but evidence for the regulation of life history traits such as fecundity and longevity have been lacking so far. In contrast, in solitary insects such as the pea aphid, the inhibition of histone acetylation and deacetylation affects development, fertility, and longevity (Kirfel, Kessel, Skaljac, Seip, \& Vilcinskas, 2020). In fact, fertility appears to be regulated by this epigenetic mark in various taxonomic groups, which includes other insects such as the planthopper Nilaparvata lugens (Zhang et al., 2018), but also mammals including mice (Ma, Pan, Montgomery, Olson, \& Schultz, 2012) and men (Wang, Gao, Li, \& Liu, 2019).

The inhibition of histone acetylation using C646 had more severe consequences on gene expression in worker fat bodies compared to the inhibition of histone deacetylation using TSA, shifting the expression of nearly 10 times as many genes. Among the most strongly downregulated genes in ants treated with the chemical inhibitors, we found interesting candidates such as the Indy protein, which has been linked to longevity in Drosophila (Rogina \& Helfand, 2013), the enzyme proclotting, which plays a role in the innate immune response (Villanueva-Segura et al., 2020), or a gene associated with juvenile hormone, which is involved in many physiological processes including aging (Yamamoto et al., 2013). We also detected seven downregulated genes associated with the fatty-acid synthesis in the queenless, C646-treated group compared to only one in the queenless control. Fatty acids are involved in the synthesis of cuticular hydrocarbons (Blomquist \& Bagnères, 2010) and reproductive and non-reproductive individuals exhibit different odors in social insects (Heinze, Stengl, \& Sledge, 2002; Monnin, 2006), including Temnothorax ants (Kleeberg, Menzel, \& Foitzik, 2017). Besides, many histone-related genes were upregulated in the group where histone acetylation was inhibited compared to our control, attesting to the efficiency of our treatment on a molecular level. Nevertheless, Chromatin Immunoprecipitation sequencing (ChIP-sequencing) would be our next logical step to both confirm changes in acetylation on the histone level and to associate histone acetylation patterns with the expression of genes of interest.

Between $33 \%$ and $40 \%$ of all differentially expressed genes were commonly upregulated in the queenright control and the queenless group treated with the inhibitor of histone acetylation C646, both groups containing workers with less developed ovaries. Among those overlapping genes, many were involved in the regulation of the circadian rhythm. On one side, this is in line with a previous study in another Temnothorax species where the use of C646 led to the loss of the ability to adjust to novel daily rhythms (Libbrecht et al., 2020). On the other side, queen presence has been found to affect worker and colony activity in the honeybee (Grodzicki, Piechowicz, \& Caputa, 2020; Moritz \& Sakofsky, 1991), which could explain the upregulation of circadian rhythm-related genes in workers from colonies where the queen remained. We could alternatively speculate that worker sterility is maintained by the upregulation of genes, which are then downregulated when workers become fecund following queen removal, explaining the large overlap of genes between our two groups.

The candidate gene "transketolase-like protein 2 isoform X2"was commonly downregulated in infertile workers and workers treated with the two chemical inhibitors, despite the relatively small number of genes (i.e., five) in the two lists of commonly downregulated genes. Transketolases are enzymes involved in the nonoxidative part of the pentose phosphate pathway (PPP) in all living organisms (Schenk et al., 1998). They are known to maintain low levels of reactive oxygen species (ROS) and are thus used in cancer treatment (Xu et al., 2016) and parasitic disease control (Kovarova et al., 2018). More broadly, antioxidant production has been positively associated with lifespan in the fruit fly (Orr \& Sohal, 1994) and the nematode Caenorhabditis elegans (Sampayo, Olsen, \& Lithgow, 2003). In our study, this gene was always upregulated in fertile ants, providing first mechanistic insights into how fecund ant workers may be able to live longer, and reinforcing findings showing that long-lived ant queens invest more in antioxidant production with age (Negroni et al., 2019).

\section{Conclusions}

The experimental use of chemical inhibitors of histone acetylation and deacetylation allowed us to show 
that this epigenetic mark might be required for workers to dynamically shift their physiology following queen removal. Our manipulation did not only affect life history traits such as fecundity, but also shifted the expression of genes with fecundity, immunity, and longevity functionalities. Our results thus provide insights into the molecular regulation of fecundity and longevity in social insects, which are prime examples of phenotypic plasticity.

\section{Acknowledgements}

We thank the Deutsche Forschungsgemeinschaft that funded this project with a grant to BF (DFG FE 1333/6-2) and SF (DFG FO 298/19-2) within the research unit FOR-2281. We are grateful to Matteo Negroni for his help during ant collection. We thank our technician Marion Kever for assistance during dissections and RNA extraction. We are very grateful to Marah Stoldt for her precious help with the analysis of the transcriptomic data. Thanks to the Southwestern Research Station (Portal, Arizona) for the support to obtain an ant collection permit from the Coronado National Forest.

\section{References}

Alexa, A., \& Rahnenführer, J. (2020). topGO: Enrichment analysis for gene ontology. R package version 2.40.0.

Altschul, S. F., Gish, W., Miller, W., Myers, E. W., \& Lipman, D. J. (1990). Basic local alignment search tool. Journal of Molecular Biology , 215 , 403-410. doi: 10.1016/S0022-2836(05)80360-2

Alvarado, S., Rajakumar, R., Abouheif, E., \& Szyf, M. (2015). Epigenetic variation in the Egfr gene generates quantitative variation in a complex trait in ants. Nature Communications ,6 (6513). doi: $10.1038 /$ ncomms7513

Amdam, G. V., Norberg, K., Hagen, A., \& Omholt, S. W. (2003). Social exploitation of vitellogenin. Proceedings of the National Academy of Sciences of the United States of America , 100 , 1799-1802. doi: 10.1073/pnas.0333979100

Andrew, S. (2010). FastQC: A quality control tool for high throughput sequence data. Available online at: http://www.bioinformatics.babraham.ac.uk/projects/fastqc/.

Bates, D., Mächler, M., Bolker, B. M., \& Walker, S. C. (2015). Fitting linear mixed-effects models using lme4. Journal of Statistical Software , 67 (1). doi: 10.18637/jss.v067.i01

Beekman, M., \& Oldroyd, B. P. (2008). When workers disunite: Intraspecific parasitism by eusocial bees. Annual Review of Entomology , 53 , 19-37. doi: 10.1146/annurev.ento.53.103106.093515

Blomquist, G. J., \& Bagnères, A.-G. (2010). Insect hydrocarbons: Biology, biochemistry, and chemical ecology . Cambridge University Press.

Bolger, A. M., Lohse, M., \& Usadel, B. (2014). Trimmomatic: A flexible trimmer for Illumina sequence data. Bioinformatics , 30 , 2114-2120. doi: 10.1093/bioinformatics/btu170

Bonasio, R. (2012). Emerging topics in epigenetics: Ants, brains, and noncoding RNAs. Annals of the New York Academy of Sciences ,1260 , 14-23. doi: 10.1111/j.1749-6632.2011.06363.x

Bonasio, R. (2014). The role of chromatin and epigenetics in the polyphenisms of ant castes. Briefings in Functional Genomics ,13 , 235-245. doi: 10.1093/bfgp/elt056

Bonasio, R., Zhang, G., Ye, C., Mutti, N. S., Fang, X., Qin, N., ... Liebig, J. (2010). Genomic comparison of the antsCamponotus floridanus and Harpegnathos saltator .Science , 329 , 1068-1070. doi: $10.1126 /$ science. 1192428 
Bowers, E. M., Yan, G., Mukherjee, C., Orry, A., Wang, L., Holbert, M. A., .. Cole, P. A. (2010). Virtual ligand screening of the p300/CBP histone acetyltransferase: Identification of a selective small molecule inhibitor. Chemistry and Biology , 17 , 471-482. doi: 10.1016/j.chembiol.2010.03.006

Cardoen, D., Wenseleers, T., Ernst, U. R., Danneels, E. L., Laget, D., De Graaf, D. C., ... Verleyen, P. (2011). Genome-wide analysis of alternative reproductive phenotypes in honeybee workers. Molecular Ecology , 20 , 4070-4084. doi: 10.1111/j.1365-294X.2011.05254.x

Choppin, M., Graf, S., Feldmeyer, B., Libbrecht, R., Menzel, F., \& Foitzik, S. (2021). Queen and worker phenotypic traits are associated with colony composition and environment in Temnothorax rugatulus(Hymenoptera: Formicidae), an ant with alternative reproductive strategies. Myrmecological News , 31 , 61-69. doi: 10.25849/myrmecol.news

Cini, A. (2014). Reliability of yellow bodies as indexes of egg laying activity in the primitively eusocial wasp Polistes dominula .Redia Giornale Di Zoologia , 97 , 183-188.

DeVeale, B., Brummel, T., \& Seroude, L. (2004). Immunity and aging: The enemy within? Aging Cell , 3 , 195-208. doi: 10.1111/j.1474-9728.2004.00106.x

dos Santos Conceição Lopes, B., Campbell, A. J., \& Contrera, F. A. L. (2020). Queen loss changes behavior and increases longevity in a stingless bee. Behavioral Ecology and Sociobiology ,74 (35). doi: 10.1007/s00265020-2811-8

Endler, A., Liebig, J., Schmitt, T., Parker, J. E., Jones, G. R., Schreier, P., \& Hölldobler, B. (2004). Surface hydrocarbons of queen eggs regulate worker reproduction in a social insect. Proceedings of the National Academy of Sciences of the United States of America ,101 , 2945-2950. doi: 10.1073/pnas.0308447101

Feldmeyer, B., Elsner, D., \& Foitzik, S. (2014). Gene expression patterns associated with caste and reproductive status in ants: Worker-specific genes are more derived than queen-specific ones.Molecular Ecology , 23 , 151-161. doi: 10.1111/mec.12490

Foster, K. R., \& Ratnieks, F. L. W. (2000). Facultative worker policing in a wasp. Nature , 407 , 692-693. doi: $10.1038 / 35037665$

Garschall, K., \& Flatt, T. (2018). The interplay between immunity and aging in Drosophila [version 1; referees: 2 approved].F1000Research , 7 (160). doi: 10.12688/f1000research.13117.1

Giehr, J., Senninger, L., Ruhland, K., \& Heinze, J. (2020). Ant workers produce males in queenless parts of multi-nest colonies.Scientific Reports , 10 (2152). doi: 10.1038/s41598-020-58830-w

Giehr, J., Wallner, J., Senninger, L., Ruhland, K., Krüger, T., \& Heinze, J. (2020). Substantial direct fitness gains of workers in a highly eusocial ant. Molecular Ecology , 29 , 3720-3730. doi: 10.1111/mec.15586

Glastad, K. M., Graham, R. J., Ju, L., Roessler, J., Brady, C. M., \& Berger, S. L. (2019). Epigenetic regulator CoREST controls social behavior in ants. Molecular Cell , 77 , 1-14. doi: 10.1016/j.molcel.2019.10.012

Grodzicki, P., Piechowicz, B., \& Caputa, M. (2020). The effect of the queen's presence on thermal behavior and locomotor activity of small groups of worker honey bees. Insects , 11 (464). doi: 10.3390/insects11080464

Haas, B. J., Papanicolaou, A., Yassour, M., Grabherr, M., Philip, D., Bowden, J., .. Regev, A. (2013). De novo transcript sequence reconstruction from RNA-Seq: Reference generation and analysis with Trinity. Nature Protocols , 8 (8). doi: 10.1038/nprot.2013.084.De

Hamilton, W. D. (1964). The genetical evolution of social behavior. II. Journal of Theoretical Biology , 7 , 17-52. doi: 10.4324/9780203790427-5

Hartfelder, K. (2000). Insect juvenile hormone: From "status quo" to high society. Brazilian Journal of Medical and Biological Research , 33 , 157-177. doi: 10.1590/S0100-879X2000000200003 
Hartig, F. (2020). DHARMa: residual diagnostics for hierarchical (multi-Level / mixed) regression models. R package version 0.3.1. https://CRAN.R-project.org/package=DHARMa.

Heinze, J. (2008). Hierarchy length in orphaned colonies of the antTemnothorax nylanderi . Naturwissenschaften , 95 , 757-760. doi: 10.1007/s00114-008-0375-y

Heinze, J., Hölldobler, B., \& Trenkle, S. (1995). Reproductive behavior of the ant Leptothorax (Dichothorax) pergandei .Insectes Sociaux , 42 , 309-315. doi: 10.1007/BF01240425

Heinze, J., \& Schrempf, A. (2008). Aging and reproduction in social insects - A mini-review. Gerontology , 54, 160-167. doi: 10.1159/000122472

Heinze, J., Stengl, B., \& Sledge, M. F. (2002). Worker rank, reproductive status and cuticular hydrocarbon signature in the ant,Pachycondyla cf. inversa . Behavioral Ecology and Sociobiology , 52 , 59-65. doi: $10.1007 / \mathrm{s} 00265-002-0491-1$

Herb, B. R. (2014). Epigenetics as an answer to Darwin's "special difficulty." Frontiers in Genetics , 5 (321). doi: 10.3389/fgene.2014.00321

Hölldobler, B., \& Wilson, E. O. (1990). The ants . Harvard University Press, Cambridge, Massachusetts.

Kim, D., Langmead, B., \& Salzberg, S. L. (2015). HISAT: A fast spliced aligner with low memory requirements. Nature Methods ,12 (4). doi: 10.1038/nmeth.3317

Kirfel, P., Kessel, T., Skaljac, M., Seip, M., \& Vilcinskas, A. (2020). Inhibition of histone acetylation and deacetylation enzymes affects longevity, development, and fecundity in the pea aphid (Acyrthosiphon pisum ). Archives of Insect Biochemistry and Physiology , 103 (e21614). doi: 10.1002/arch.21614

Kleeberg, I., Menzel, F., \& Foitzik, S. (2017). The influence of slavemaking lifestyle, caste and sex on chemical profiles in Temnothorax ants: Insights into the evolution of cuticular hydrocarbons. Proceedings of the Royal Society B: Biological Sciences , 284 (20162249). doi: 10.1098/rspb.2016.2249

Kohlmeier, P., Feldmeyer, B., \& Foitzik, S. (2018). Vitellogenin-like A-associated shifts in social cue responsiveness regulate behavioral task specialization in an ant. PLoS Biology, 16 (e2005747). doi: 10.1371/journal.pbio. 2005747

Kohlmeier, P., Negroni, M. A., Kever, M., Emmling, S., Stypa, H., Feldmeyer, B., \& Foitzik, S. (2017). Intrinsic worker mortality depends on behavioral caste and the queens' presence in a social insect.Science of Nature , 104 (34). doi: 10.1007/s00114-017-1452-x

Kolder, R. (2012). Pheatmap: pretty heatmaps, R package version 1.0.12 (https:// CRAN.Rproject.org/package=pheatmap).

Konrad, M., Pamminger, T., \& Foitzik, S. (2012). Two pathways ensuring social harmony. Naturwissenschaften , 99 , 627-636. doi: 10.1007/s00114-012-0943-z

Korb, J. (2016). Why do social insect queens live so long? Approaches to unravel the sociality-aging puzzle. Current Opinion in Insect Science , 16 , 104-107. doi: 10.1016/j.cois.2016.06.004

Kovarova, J., Pountain, A. W., Wildrige, D., Weidt, S., Bingaud, F., Burchmore, R. J. S., .. Barrett, M. P. (2018). Deletion of transketolase triggers a stringent metabolic response in promastigotes and loss of virulence in amastigotes of Leishmania mexicana .PLoS Pathogens , 14 (3). doi: 10.1093/nar/gks1004

Li, H., Handsaker, B., Wysoker, A., Fennell, T., Ruan, J., Homer, N., .. Sam, T. (2009). The Sequence Alignment / Map format and SAMtools. Bioinformatics , 25 (16). doi: 10.1093/bioinformatics/btp352

Libbrecht, R., Nadrau, D., \& Foitzik, S. (2020). A role of histone acetylation in the regulation of circadian rhythm in ants.IScience, 23 (100846). doi: 10.1016/j.isci.2020.100846 
Love, M. I., Huber, W., \& Anders, S. (2014). Moderated estimation of fold change and dispersion for RNA-seq data with DESeq2. Genome Biology , 15 (550). doi: 10.1186/s13059-014-0550-8

Ma, P., Pan, H., Montgomery, R. L., Olson, E. N., \& Schultz, R. M. (2012). Compensatory functions of histone deacetylase 1 (HDAC1) and HDAC2 regulate transcription and apoptosis during mouse oocyte development. Proceedings of the National Academy of Sciences of the United States of America, 109 (8). doi: 10.1073/pnas.1118403109

Majoe, M., Libbrecht, R., Foitzik, S., \& Nehring, V. (2021). Queen loss increases worker survival in leafcutting ants under paraquat-induced oxidative stress. Philosophical Transactions of the Royal Society B , 376 (20190735). doi: 10.1098/rstb.2019.0735

Maleszka, R. (2016). Epigenetic code and insect behavioural plasticity.Current Opinion in Insect Science, 15 , 45-52. doi: 10.1016/j.cois.2016.03.003

Marshall, H., Lonsdale, Z. N., \& Mallon, E. B. (2019). Methylation and gene expression differences between reproductive and sterile bumblebee workers. Evolution Letters , 3 (5), 485-499. doi: 10.1002/evl3.129

Matsuura, K., Himuro, C., Yokoi, T., Yamamoto, Y., Vargo, E. L., \& Keller, L. (2010). Identification of a pheromone regulating caste differentiation in termites. Proceedings of the National Academy of Sciences of the United States of America , 107 (29). doi: 10.1073/pnas.1004675107

Monnin, T. (2006). Chemical recognition of reproductive status in social insects. Finnish Zoological and Botanical Publishing Board ,43 , 515-530.

Monnin, T., \& Peeters, C. (1999). Dominance hierarchy and reproductive conflicts among subordinates in a monogynous queenless ant.Behavioral Ecology , 10 (3). doi: 10.1093/beheco/10.3.323

Monroy Kuhn, J. M., \& Korb, J. (2016). Editorial overview: Social insects: aging and the re-shaping of the fecundity/longevity trade-off with sociality. Current Opinion in Insect Science, 16 (vii-x). doi: 10.1016/j.cois.2016.06.002

Morandin, C., Havukainen, H., Kulmuni, J., Dhaygude, K., Trontti, K., \& Helanterä, H. (2014). Not only for egg yolk-functional and evolutionary insights from expression, selection, and structural analyses ofFormica ant vitellogenins. Molecular Biology and Evolution , 31 (8). doi: 10.1093/molbev/msu171

Moritz, R. F. A., \& Sakofsky, F. (1991). The role of the queen in circadian rhythms of honeybees (Apis mellifera L.).Behavioral Ecology and Sociobiology , 29 , 361-365. doi: 10.1007/BF00165961

Negroni, M. A., Foitzik, S., \& Feldmeyer, B. (2019). Long-lived Temnothorax ant queens switch from investment in immunity to antioxidant production with age. Scientific Reports , 9 , 7270. doi: 10.1038/s41598$019-43796-1$

Negroni, M. A., Macit, M. N., Stoldt, M., Feldmeyer, B., \& Foitzik, S. (2021). Molecular regulation of lifespan extension in fertile ant worker. Philosophical Transactions of the Royal Society B ,376 (20190736). doi: $10.1098 /$ rstb.2019.0736

Negroni, M. A., Segers, F. H. I. D., Vogelweith, F., \& Foitzik, S. (2020). Immune challenge reduces gut microbial diversity and triggers fertility-dependent gene expression changes in a social insect.BMC Genomics , 21 (816). doi: 10.1186/s12864-020-07191-9

Okonechnikov, K., Conesa, A., \& García-Alcalde, F. (2016). Qualimap 2: Advanced multi-sample quality control for high-throughput sequencing data. Bioinformatics , 32 (2). doi: 10.1093/bioinformatics/btv566

Oldroyd, B. P., Halling, L. A., Good, G., Wattanachaiyingcharoen, W., Barton, A. B., Nanork, P., ... Ratnieks, F. L. W. (2001). Worker policing and worker reproduction in Apis cerana . Behavioral Ecology and Sociobiology , 50 (4). doi: 10.1007/s002650100376 
Orr, W. C., \& Sohal, R. S. (1994). Extension of life-span by overexpression of superoxide dismutase and catalase in Drosophila melanogaster . Science , 263 (5150). doi: 10.1126/science.8108730

Parker, J. D. (2010). What are social insects telling us about aging? Myrmecological News , 13 , 103-110.

Peeters, C., \& Tinaut, A. (2014). Reconstructing life history in ants: Ergatoid queens, facultative monogyny, anddependent colony foundation in Monomorium algiricum (Hymenoptera: Formicidae).Myrmecological News , 20 , 77-85. doi: 10.5281/zenodo.844253

Pertea, M., Pertea, G. M., Antonescu, C. M., Chang, T.-C., Mendell, J. T., \& Salzberg, S. L. (2015). StringTie enables improved reconstruction of a transcriptome from RNA-seq reads. Nature Biotechnology ,33 (3). doi: $10.1038 /$ nbt. 3122

Quevillon, E., Silventoinen, V., Pillai, S., Harte, N., Mulder, N., Apweiler, R., \& Lopez, R. (2005). InterProScan: Protein domains identifier. Nucleic Acids Research, 33, W116-W120. doi: 10.1093/nar/gki442

R Core Team. (2020). R: a language and environment for statistical computing. R foundation for statistical computing, Vienna, Austria. URL https://www.R-project.org/.

Rogina, B., \& Helfand, S. L. (2013). Indy mutations andDrosophila longevity. Frontiers in Genetics , 4 (47). doi: $10.3389 /$ fgene.2013.00047

Ruhland, F., Moulin, M., Choppin, M., Meunier, J., \& Lucas, C. (2020). Reproductives and eggs trigger worker vibration in a subterranean termite. Ecology and Evolution , 00 , 1-7. doi: 10.1002/ece3.6325

Rüppell, O., Heinze, J., \& Hölldobler, B. (1998). Size-dimorphism in the queens of the North American ant Leptothorax rugatulus(Emery). Insectes Sociaux , 45 , 67-77. doi: 10.1007/s000400050069

Rüppell, O., Heinze, J., \& Hölldobler, B. (2001). Alternative reproductive tactics in the queen-size-dimorphic ant Leptothorax rugatulus (Emery) and their consequences for genetic population structure. Behavioral Ecology and Sociobiology , 50 , 189-197. doi: 10.1007/s002650100359

Sampayo, J. N., Olsen, A., \& Lithgow, G. J. (2003). Oxidative stress inCaenorhabditis elegans : Protective effects of superoxide dismutase/catalase mimetics. Aging Cell , 2 , 319-326. doi: 10.1046/j.14749728.2003.00063.x

Schenk, G., Duggleby, R. G., \& Nixon, P. F. (1998). Properties and functions of the thiamin diphosphate dependent enzyme transketolase.International Journal of Biochemistry and Cell Biology ,30 , 1297-1318. doi: 10.1016/S1357-2725(98)00095-8

Schrempf, A., Giehr, J., Röhrl, R., Steigleder, S., \& Heinze, J. (2017). Royal Darwinian demons: Enforced changes in reproductive efforts do not affect the life expectancy of ant queens. The American Naturalist, 189 (4). doi: $10.1086 / 691000$

Seehuus, S. C., Norberg, K., Gimsa, U., Krekling, T., \& Amdam, G. V. (2006). Reproductive protein protects functionally sterile honey bee workers from oxidative stress. Proceedings of the National Academy of Sciences of the United States of America , 103 (4). doi: 10.1073/pnas.0502681103

Simola, D. F., Graham, R. J., Brady, C. M., Enzmann, B. L., Desplan, C., Ray, A., ... Berger, S. L. (2016). Epigenetic (re)programming of caste-specific behavior in the ant Camponotus floridanus .Science, 351 (6268). doi: 10.1126/science.aac6633

Simola, D. F., Ye, C., Mutti, N. S., Doleza, K., Bonasio, R., Liebig, J., .. Berger, S. L. (2013). A chromatin link to caste identity in the carpenter ant. Genome Research , 23 , 486-496. doi: 10.1101/gr.148361.112.486

Smith-Unna, R., Boursnell, C., Patro, R., Hibberd, J. M., \& Kelly, S. (2016). TransRate: Referencefree quality assessment of de novo transcriptome assemblies. Genome Research , 26 , 1134-1144. doi: $10.1101 /$ gr.196469.115 
Spannhoff, A., Kim, Y. K., Raynal, N. J. M., Gharibyan, V., Su, M. B., Zhou, Y. Y., .. Bedford, M. T. (2011). Histone deacetylase inhibitor activity in royal jelly might facilitate caste switching in bees. EMBO Reports , 12 (3). doi: 10.1038/embor.2011.9

Taylor, B. A., Cini, A., Sumner, S., Wyatt, C. D. R., \& Reuter, M. (2021). The molecular basis of socially mediated phenotypic plasticity in a eusocial paper wasp. Nature Communications , 12 (775). doi: $10.1038 / \mathrm{s} 41467-021-21095-6$

Vaiserman, A. (2015). Developmental epigenetic programming of caste-specific differences in social insects: An impact on longevity.Current Aging Science , 7 , 176-186. doi: 10.2174/1874609807666141129173749

Vaiserman, A., Lushchak, O. V., \& Koliada, A. K. (2018). Epigenetics of longevity in social insects. In Epigenetics of Aging and Longevity (pp. 271-289). Elsevier Inc. doi: 10.1016/b978-0-12-811060-7.00013-9

Valanne, S., Wang, J.-H., \& Rämet, M. (2011). The DrosophilaToll signaling pathway. The Journal of Immunology , 186 , 649-656. doi: 10.4049/jimmunol.1002302

Van Oystaeyen, A., Oliveira, R. C., Holman, L., van Zweden, J. S., Romero, C., Oi, C. A., ... Wenseleers, T. (2014). Conserved class of queen pheromones stops social insect workers from reproducing.Science, 343 , 287-290. doi: 10.1126/science.1244899

Villanueva-Segura, O. K., Gómez-Govea, M. A., Garza-Veloz, I., González-Alvarez, R., Carrillo-Gaytán, D., Ramírez-Valles, E. G., .. Guzmán-Velasco, A. (2020). Molecular description of proclotting enzyme: a power tool for insect biological control of Aedes aegypti L. Southwestern Entomologist , 45 (3). doi: $10.3958 / 059.045 .0312$

Wang, T., Gao, H., Li, W., \& Liu, C. (2019). Essential role of histone replacement and modifications in male fertility. Frontiers in Genetics , 10 (962). doi: 10.3389/fgene.2019.00962

Wickham, H. (2010). ggplot2: Elegant graphics for data analysis. Journal of Statistical Software , 35 (1). doi: 10.1007/978-0-387-98141-3

Wojciechowski, M., Lowe, R., Maleszka, J., Conn, D., Maleszka, R., \& Hurd, P. J. (2018). Phenotypically distinct female castes in honey bees are defined by alternative chromatin states during larval development.Genome Research , 28 , 1532-1542. doi: 10.1101/gr.236497.118

Wurm, Y., Wang, J., \& Keller, L. (2010). Changes in reproductive roles are associated with changes in gene expression in fire ant queens. Molecular Ecology , 19 , 1200-1211. doi: 10.1111/j.1365-294X.2010.04561.x

Xu, I. M. J., Lai, R. K. H., Lin, S. H., Tse, A. P. W., Chiu, D. K. C., Koh, H. Y., .. Ng, I. O. L. (2016). Transketolase counteracts oxidative stress to drive cancer development. Proceedings of the National Academy of Sciences of the United States of America ,113 , E725-E734. doi: 10.1073/pnas.1508779113

Yamamoto, R., Bai, H., Dolezal, A. G., Amdam, G., \& Tatar, M. (2013). Juvenile hormone regulation of Drosophila aging. BMC Biology , 11 (85). doi: 10.1186/1741-7007-11-85

Yan, H., Bonasio, R., Simola, D. F., Liebig, J., Berger, S. L., \& Reinberg, D. (2015). DNA methylation in social insects: How epigenetics can control behavior and longevity. Annual Review of Entomology ,60 , 435-452. doi: 10.1146/annurev-ento-010814-020803

Yoshidas, M. (1990). Potent and specific inhibition of mammalian histone deacetylase both in vivo and in vitro Trichostatin A*. The Journal of Biological Chemistry , 265 (28). doi: 10.1016/S0021-9258(17)44885-X.

Zhang, J. L., Yuan, X. B., Chen, S. J., Chen, H. H., Xu, N., Xue, W. H., .. Xu, H. J. (2018). The histone deacetylase NIHDAC1 regulates both female and male fertility in the brown planthopper,Nilaparvata lugens . Open Biology , 8 (180158). doi: 10.1098/rsob.180158

\section{Data Accessibility}


Scripts and data sets for both the statistical analysis of worker number and fecundity and the gene expression analysis are available in a shared Google Drive folder at:https://drive.google.com/drive/folders/1f8pA772Yaogk8i-A1xiAiBRiFAsCohX8?usp=sharing. Raw reads for the transcriptomic analysis and associated metadata have been uploaded on the Short Read Archive (SRA) from NCBI under the BioProject PRJNA717637 (http://www.ncbi.nlm.nih.gov/bioproject/717637) and will be available after publication.

\section{Author Contributions}

$\mathrm{MC}, \mathrm{BF}$, and SF collected the ant colonies and designed the study. MC conducted the experiment and analyzed the transcriptomic data. All three authors contributed to data interpretation, writing, and approval of the final manuscript.

\section{Tables and Figures}

\begin{tabular}{llll}
\hline Group name & Manipulation & Treatment & Colonies \\
\hline Queenright & No queen removal & DMSO & $\mathrm{N}=18 / 4$ \\
Queenless & Queen removal & DMSO & $\mathrm{N}=18 / 5$ \\
Queenless+C646 & Queen removal & DMSO+C646 & $\mathrm{N}=18 / 7$ \\
Queenless+TSA & Queen removal & DMSO+TSA & $\mathrm{N}=18 / 4$ \\
Queenless+C646/TSA & Queen removal & DMSO+C646/TSA & $\mathrm{N}=18 / 0$ \\
\hline
\end{tabular}

Table 1. Group name, manipulation, treatment, and sample sizes $(\mathrm{N}=$ colonies at start $/ \mathrm{N}=$ colonies for RNA-sequencing of fat body samples) for each experimental group.

Note: The queenless control was used as a reference in all comparisons.
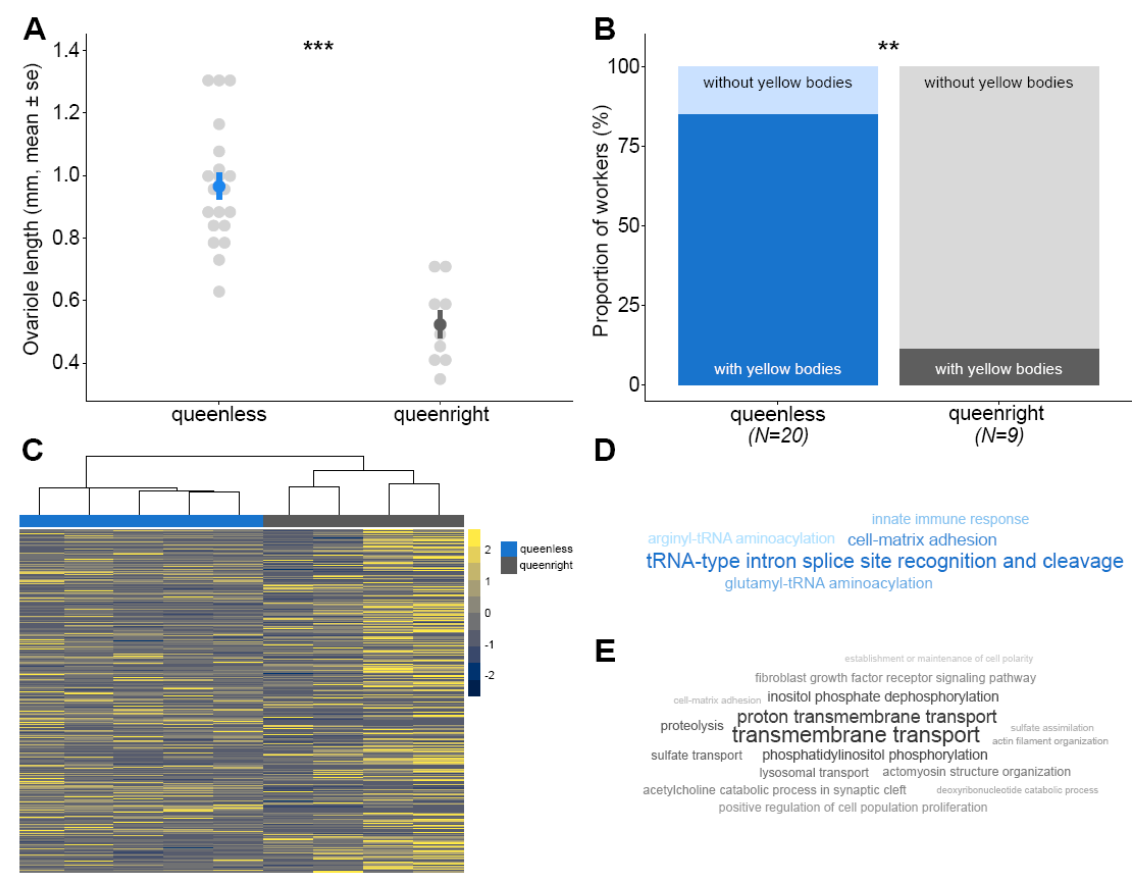

Figure 1. (A) Effect of queen removal on ovariole length and (B) the proportion of workers with yellow 
bodies in their ovaries at the end of the experiment at week 6 . Levels of significance are indicated as follows: ${ }^{* *} \mathrm{p}<0.01$ and ${ }^{* * *} \mathrm{p}<0.001$. (C) heatmap showing the expression levels of the differentially expressed genes between the groups "queenless" (control, blue) and "queenright" (grey) and the clustering of samples per group. (D) and (E) word clouds showing the overrepresented functions associated with the genes downregulated (top, blue) and upregulated (bottom, grey) in the group "queenright". In the word clouds p-value significance is positively correlated with the size and shade darkness of the word.
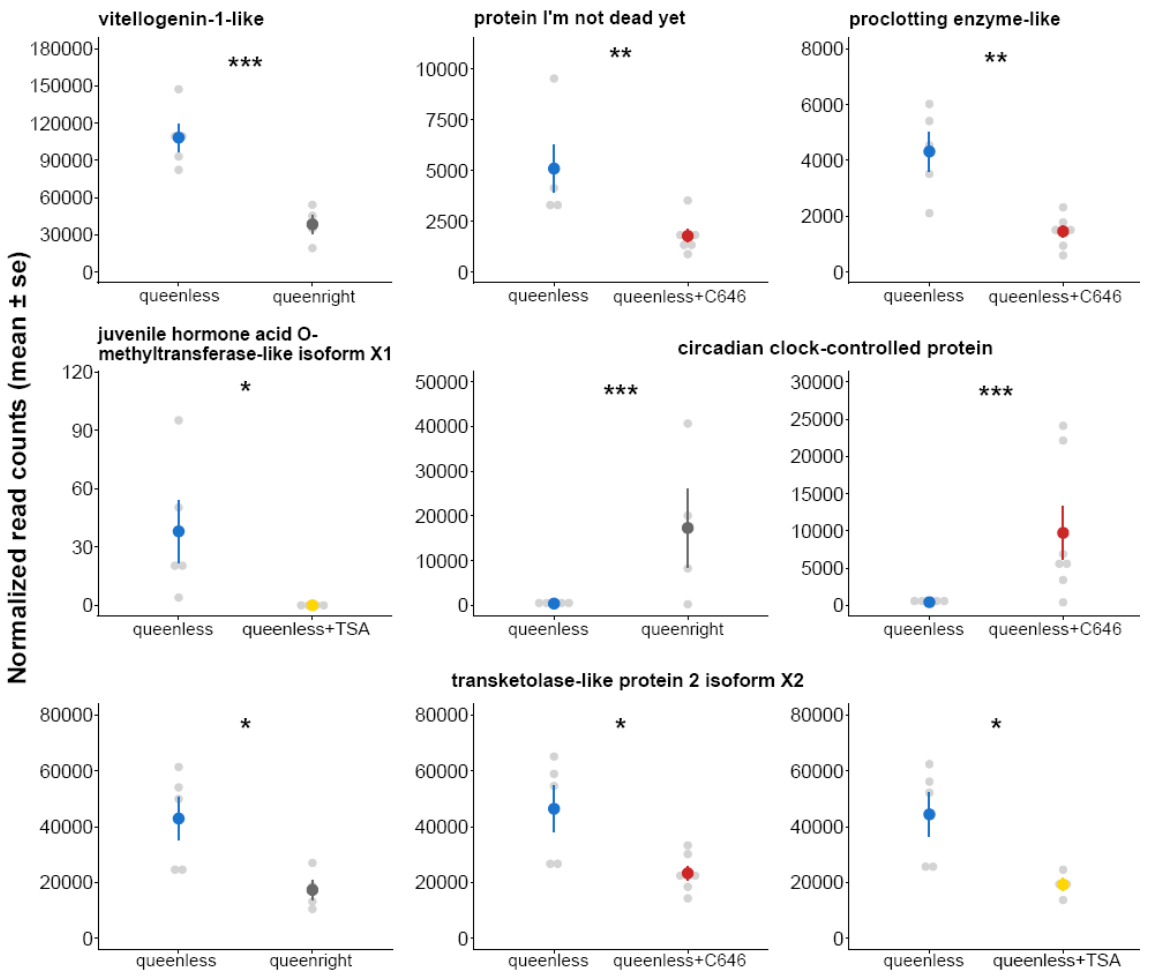

Figure 2. First row: expression levels of the genes "vitellogenin-1-like" ( $<<0.001)$ downregulated in the group "queenright" compared to the group "queenless" (control), "protein I'm not deat yet" (p = 0.004) and "proclotting enzyme-like" ( $\mathrm{p}=0.001)$ downregulated in the group "queenless+C646" compared to the group "queenless" (control). Second row: expression levels of the genes "juvenile hormone acid-Omethyltransferase-like isoform X1" ( $\mathrm{p}=0.01)$ downregulated in the group "queenless+TSA" compared to the group "queenless" (control) and the gene "circadian clock-controlled protein" (pQR $<0.001$ and pC646 < 0.001 ) commonly downregulated in the groups "queenright" and "queenless+C646" compared to "queenless" (control). Third row: expression levels of the gene "transketolase-like protein 2 isoform X2" (pQR $=0.036$, pC646 $=0.031$ and pTSA $=0.047)$ commonly downregulated in the groups "queenright", "queenless+C646" and "queenless+TSA" compared to "queenless" (control). Levels of significance are indicated as follows: * $\mathrm{p}<0.05,{ }^{* *} \mathrm{p}<0.01$ and ${ }^{* * *} \mathrm{p}<0.001$. 

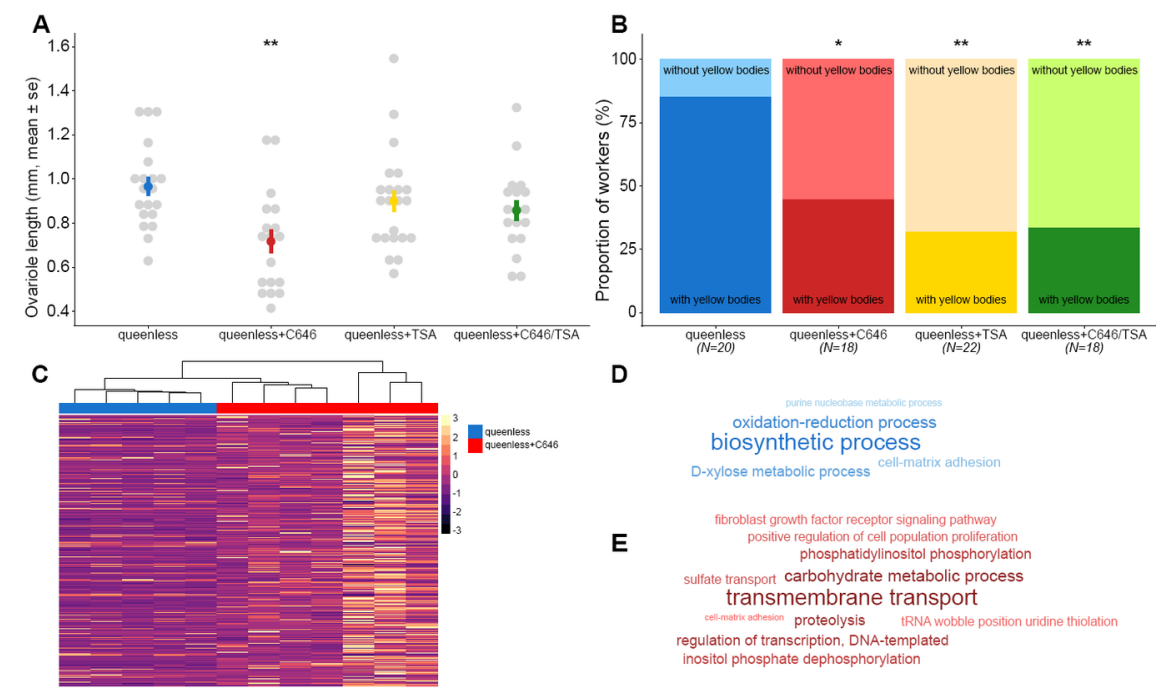

D

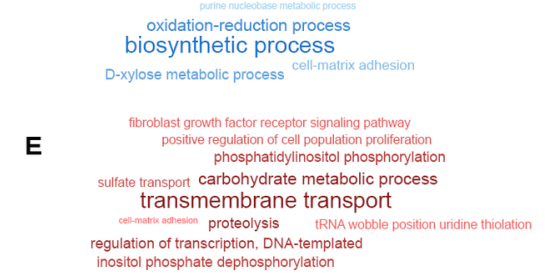

Figure 3. (A) Effect of treatment on ovariole length and (B) the proportion of workers with yellow bodies in their ovaries at the end of the experiment at week 6 . Levels of significance are indicated as follows: ${ }^{*} \mathrm{p}<0.05$ and ${ }^{* *} \mathrm{p}<0.01$. (C) heatmap showing the expression levels of the differentially expressed genes between the groups "queenless" (control, blue) and "queenless+C646" (red) and the clustering of samples per group. (D) and (E) word clouds showing the overrepresented functions associated with the genes downregulated (top, blue) and upregulated (bottom, red) in the group "queenless+C646". In the word clouds p-value significance is positively correlated with the size and shade darkness of the word. 

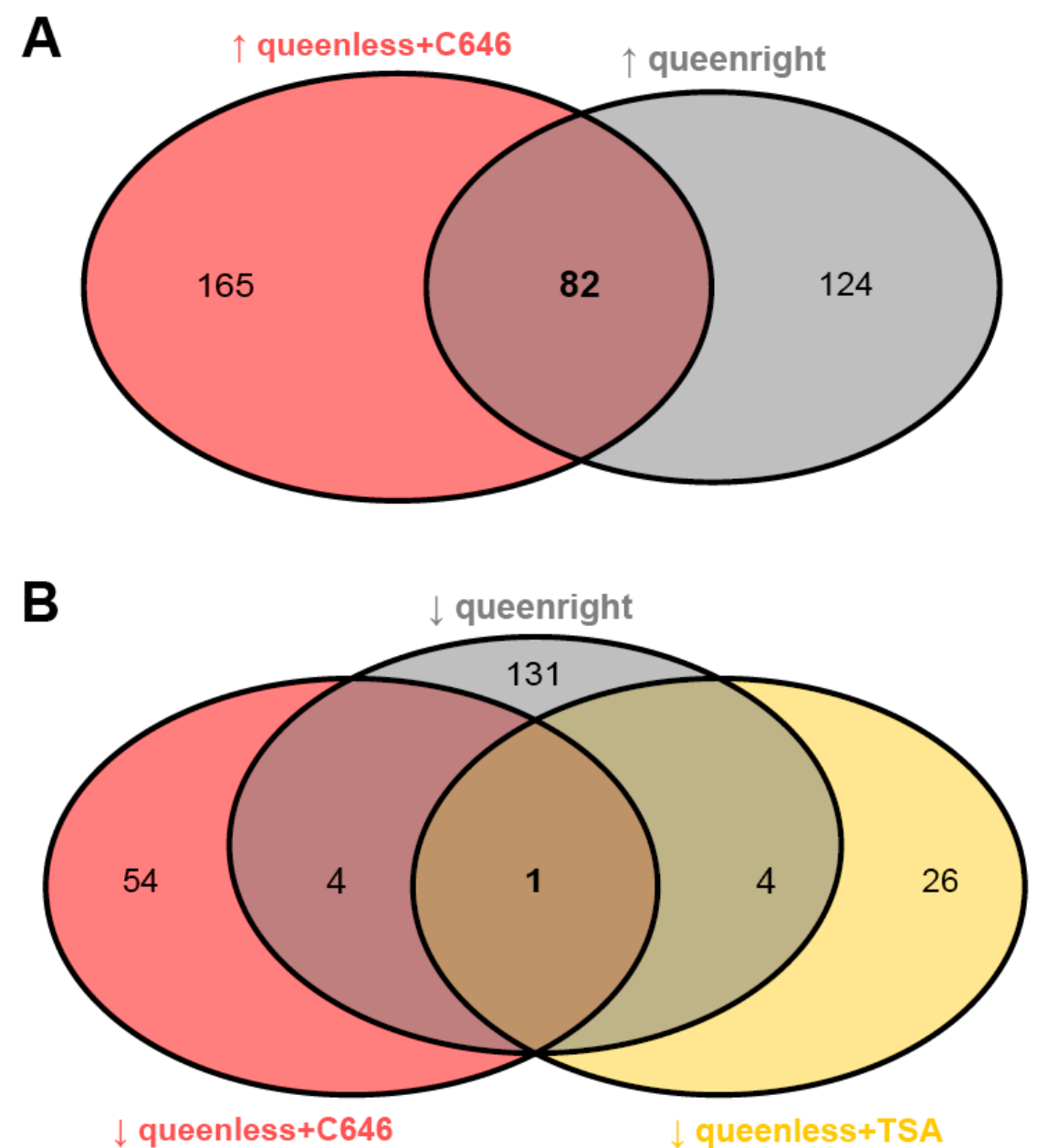

Figure 4 . (A) Venn diagram showing the number of genes singly upregulated in the groups "queenless+C646" (165) and "queenright" (124) and the number of genes commonly upregulated between these two groups (82). (B) Venn diagram showing the number of genes singly downregulated in the groups "queenless+C646" (54), "queenright" (131) and "queenless+TSA" (26), the number of genes commonly downregulated between "queenright" and "queenless+C646" (4) or "queenless+TSA" (4) and the number of genes commonly downregulated between all three groups (1). 